\title{
Gene networks of type 2 diabetes and Alzheimer's disease. Reconstruction and analysis
}

\author{
Zamyatin V. ${ }^{1,2 *}$, Mustafin Z.S. ${ }^{1,2}$, Matushkin Y.G. ${ }^{1,2}$, Klimontov V.V. ${ }^{1,2}$, Lashin S.A. ${ }^{1,2}$ \\ ${ }^{1}$ Institute of Cytology and Genetics, SB RAS, Novosibirsk, Russia \\ ${ }^{2}$ Novosibirsk State University, Novosibirsk, Russia \\ *e-mail: zamyatin@bionet.nsc.ru
}

Key words: gene networks, SNP, T2D, diabetes, AD, Alzheimer's disease

Motivation and Aim: Type 2 diabetes (T2D) and Alzheimer's disease (AD) are multifactorial diseases caused by a combination of genetic and environmental factors. Today, systems biology approaches and network approaches are powerful tools for studying multifactor diseases. They are based on knowledge of physical and/or functional interactions between molecules, usually represented as networks. Such networks not only report connections between individual nodes, but also implicitly organize highlevel cellular connections. In this study, we consider networks of transcriptional regulation, protein-protein interactions, and functional connectivity, leading them to the general meaning of gene networks - molecular-genetic systems ensuring the phenotypic properties development of organisms based on genetic information [1].

Methods and Algorithms: Based on an analysis of literature data, as well as a number of databases, sets of genes associated with AD and T2D were formed. Among them, transcription factors (TF) were identified. HOCOMOCO database was used for search the binding sites of TF in 5'-noncoding regions of genes. The phylostratigraphic age and the divergence index were calculated for each gene using Orthoscape software [2]. The intrapopulation variability was estimated using the data on the SNP of the project " 1000 human genomes". Based on the transcriptomes found, differential gene expression was analyzed. Based on the "raw" data from type 2 diabetes mellitus and Alzheimer's disease researches, a search was made for genome-wide associations. Gene networks were reconstructed using Cytoscape based on interaction data extracted from GeneMANIA and StringDB.

Results: After applying the information obtained earlier, an analysis of the gene networks was carried out. Were identified regulatory circuits, violations in the elements of which can lead to the diseases. They contain new genes that were not previously associated with T2D.

Conclusion: Analysis of gene networks is an effective tool for a comprehensive theoretical study of human diseases and it can be used to not only search for and prioritize genes associated with diseases, but also to explain the molecular genetic mechanisms behind them.

Acknowledgements: The study was supported by the Project 0324-2018-0021 SB RAS.

\section{References}

1. Introduction to informational biology and bioinformatics: study guide: eds N.A. Kolchanov, O.V. Vishnevsky, D.P. Furman. - Novosibirsk, 2015;3 (in Russian).

2. Mustafin Z.S. et al. Orthoscape: A cytoscape application for grouping and visualization KEGG based gene networks by taxonomy and homology principles. BMC Bioinformatics. 2017;18. 